\title{
地理来源与生物化学属性对泥炭地植物残体分解 的影响
}

\author{
刘媛媛 $1,2,3$ 马进泽 $1,2,3$ 卜兆君 ${ }^{1,2,3^{*}}$ 王升忠 $1,2,3^{*}$ 张雪冰 ${ }^{1}$ 张婷玉 $^{1}$ 刘莎莎 $^{1,2,3}$ \\ 付 虎 ${ }^{1}$ 康 媛 $1,2,3$
}

${ }^{1}$ 东北师范大学地理科学学院泥炭沼泽研究所, 长春 130024 ; ${ }^{2}$ 国家环境保护湿地生态与植被恢复重点实验室, 长春 130024 ; ${ }^{3}$ 长白山湿地生态过程与 环境变化吉林省重点实验室, 长春 130024

\begin{abstract}
摘 要 不同地理来源的泥炭地植物残体在同一环境中的分解速率一直缺乏比较研究。该研究沿纬度梯度, 选择大九湖、哈 泥和满归 3 处泥炭地, 以三地的 10 种植物为分解材料, 使用分解袋包装, 埋藏于长白山哈泥泥炭地, 开展为期 1 年的分解实验, 研究地理来源及生物化学属性对泥炭地植物残体分解的影响。结果表明, 如不考虑物种差异, 从总体上看, 随着纬度增加, 3 处泥炭地植物残体的初始氮 $(\mathrm{N})$ 含量下降, 初始木质素含量、碳氮比 $(\mathrm{C} / \mathrm{N})$ 和木质素 $/ \mathrm{N}$ 上升。经一年分解后残体分解速率因植 物类群不同而不同, 桦木属(Betula) 和薹草属(Carex)植物残体的干质量损失率均接近 $50 \%$, 远大于泥炭藓属(Sphagnum)植物 (约为10\%)。3处来源地植物残体干质量损失率总体上无差异, 但比较同种植物残体发现, 来自中纬度泥炭地哈泥的中位泥炭 蘚(S. magellanicum)的干质量损失率(19\%)远高于来自高纬度泥炭地满归的 $(9 \%)$ 。制约残体分解的因素因植物类群不同而不 同, 残体初始总酚 $/ \mathrm{N}$ 是决定属间残体干质量损失率差异的重要指标。䔔草属植物初始 $\mathrm{N}$ 含量和 $\mathrm{C} / \mathrm{N}$ 与残体分解速率、泥炭藓 属植物初始Klason木质素含量和总酚/ $\mathrm{N}$ 与残体分解速率均呈正相关关系。该研究一定程度上表明, 若以纬度降低指代气候变 暖, 当前持续的气候变暖可能通过改变高纬度泥炭地的植物组成和植物的生物化学属性, 来改变植物残体分解速率, 进而影 响泥炭地的碳汇功能。
\end{abstract}

关键词 纬度梯度格局; 植物功能群; 泥炭地; 生物化学品质

刘媛媛, 马进泽, 卜兆君, 王升忠, 张雪冰, 张婷玉, 刘莎莎, 付彪, 康媛 (2018). 地理来源与生物化学属性对泥炭地植物残体分解的影响. 植物生态 学报, 42, 713-722. DOI: 10.17521/cjpe.2018.0029

\section{Effect of geographical sources and biochemical traits on plant litter decomposition in a peatland}

LIU Yuan-Yuan ${ }^{1,2,3}$, MA Jin-Ze ${ }^{1,2,3}$, BU Zhao-Jun ${ }^{1,2,3^{*}}$, WANG Sheng-Zhong ${ }^{1,2,3^{*}}$, ZHANG Xue-Bing ${ }^{1}$, ZHANG Ting-Yu ${ }^{1}$, LIU Sha-Sha ${ }^{1,2,3}$, FU Biao ${ }^{1}$, and KANG Yuan ${ }^{1,2,3}$

${ }^{1}$ Institute for Peat and Mire Research, School of Geographical Science, Northeast Normal University, Changchun 130024, China; ${ }^{2}$ Key Laboratory for Wetland Conservation and Vegetation Restoration, Ministry of Environmental Protection, Changchun 130024, China; and Jilin Provincial Key Laboratory for Wetland Ecological Processes and Environmental Change in the Changbai Mountains, Changchun 130024, China

\section{Abstract}

Aims Few comparative studies have been conducted on the decomposition of the plant litters from different geographical sources in the same site. We aimed to understand the effect of geographical sources and biochemical traits of peatland plants on litter decomposition.

Methods Along a latitudinal gradient, we collected plant materials from three peatlands, Dajiuhu, Hani and Mangui, to carry out a one-year decomposition experiment with litter bags in Hani Peatland, Changbai Mountains. Important findings When species identity was not considered, we found that overall initial nitrogen $(\mathrm{N})$ content decreased while initial lignin content, carbon nitrogen ratio $(\mathrm{C} / \mathrm{N})$ and lignin/ $\mathrm{N}$ increased with latitude in the litters from 3 peatlands. Litter decomposition differed with plant functional groups. After one year of decomposition, dry mass loss of both birch and sedge (ca. 50\%) was higher than that of peat mosses (ca. 10\%). No significant difference was observed in litter dry mass loss among different geographical sources. However, dry mass loss of Sphagnum magellanicum from the middle latitudinal peatland (19\%) was higher than that from the high latitudinal

收稿日期Received: 2018-01-25 接受日期Accepted: 2018-07-10

基金项目：国家自然科学基金(41371103、41471043和41601085)和国家重点研发计划(2016YFC0500407)。Supported by the National Natural Science Foundation of China (41371103, 41471043 and 41601085), and the National Key Research and Development Program of China (2016YFC0500407).

* 通信作者Corresponding author (Bu ZJ: buzhaojun@nenu.edu.cn; Wang SZ: szwang@nenu.edu.cn) 
site (9\%). The factors affecting litter decomposition differed among plant functional groups. Initial total phenolics/N was the important factor to determine the difference in litter dry mass loss among the 3 genera. The initial $\mathrm{N}$ content and $\mathrm{C} / \mathrm{N}$, and Klason lignin content and total phenolics/ $\mathrm{N}$ were positively related to litter decomposition of Carex and Sphagnum, respectively. If the decrease in latitude is used to indicate climate warming, to some extent, our study suggests that current climate warming, by changing the plant composition and biochemical traits, may alter litter decomposition and even carbon accumulation in high latitudinal peatlands.

Key words latitudinal gradient pattern; plant functional group; peatland; biochemical quality

Liu YY, Ma JZ, Bu ZJ, Wang SZ, Zhang XB, Zhang TY, Liu SS, Fu B, Kang Y (2018). Effect of geographical sources and biochemical traits on plant litter decomposition in a peatland. Chinese Journal of Plant Ecology, 42, 713-722. DOI: 10.17521/cjpe.2018.0029

泥炭地是有机物生产远大于分解的生态系统, 大量的植物死亡后，缓慢分解，长期累积形成泥炭。 全球来看, 泥炭地主要分布于北方气候区。北方泥 炭地虽仅占全球陆地面积的3\%,却存储了全球土 壤大约 $1 / 3$ 的碳, 相当于大气 $\mathrm{CO}_{2}$ 中碳储量的一半, 在全球碳循环中发挥着十分重要的作用(Rydin \& Jeglum, 2013)。

通常认为, 较低的温度、酸性条件、微生物多 样性低以及植物残体自身耐分解的化学品质特征, 决定北方泥炭地的植物残体分解速率普遍很低 (Moore \& Basiliko, 2006; Rydin \& Jeglum, 2013)。大 多数研究认为, 植物残体的分解速率与残体的氮 (N)(Johnson \& Damman, 1993; Müller et al., 2003; Stubbs et al., 2009; 王行等, 2018 )和磷(P)等营养元 素含量正相关, 与木质素、纤维素和总酚含量负相 关(Dyer et al., 1990; 王瑾和黄建辉, 2001)。因此, 残 体分解有难易差异, 残体的质量有优劣之分, 甚至 一些指标间的化学计量比如碳氮比 $(\mathrm{C} / \mathrm{N})$ 和木质素 $/ \mathrm{N}$ 是表明残体品质或易分解程度的良好指标, 其数值 越高, 植物残体将越难分解(Melillo et al., 1982; Aerts, 1997; Hobbie, 2008; 欧阳林梅等, 2013; Zhang et al., 2013), 即残体的分解速率通常与 $\mathrm{C} / \mathrm{N}$ 、木质素 $/ \mathrm{N}$ 呈负相关关系。

大尺度的地理环境差异影响植物残体的分解。 在中、北美洲跨越热带、温带和寒带的对比实验研 究表明, 实验周期内热带分解常数最大, 温带和寒 带相似, 但若考虑有效时间(度日超过 $0{ }^{\circ} \mathrm{C}$ ), 则寒 带分解常数远高于热带和温带(Irons et al., 1994)。当 对残体分解纬度梯度格局的机制深入分析时, Berg 等(1993)发现, 蒸散发是决定从亚热带至亚北极近 $40^{\circ}$ 纬距范围内残体分解的最重要因素。此外, 植物 生活型对残体分解的大尺度地理格局影响很大, Körner (1989)的研究中, 草本植物更容易表现出清
晰的地理格局, 而常绿木本植物则不然。事实上, 地 理环境差异可影响植物生物化学属性和残体品质。 例如, 对欧洲来自 9 个山区150种植物的比较研究显 示, 草本植物叶片的 $\mathrm{N}$ 含量随海拔和纬度的增加而 增加(Körner, 1989); 在我国东部地区, 森林乔木的 叶片中, $\mathrm{N}$ 和P含量均随纬度增加而增加(Chen et al., 2013)。来自泥炭地的实验结果恰恰相反, Dorrepaal 等(2005)在欧洲沿纬度梯度的分解实验发现，70种 实验植物存在随着纬度增加 $\mathrm{N}$ 含量下降、 $\mathrm{C} / \mathrm{N}$ 增加的 趋势。

迄今为止, 不同纬度来源植物残体在同一地理 环境中分解的比较研究还较少, 而在泥炭地方面, 研究多集中于水淹厌氧、贫营养、较强酸性等对分 解及碳累积的直接贡献(Rydin \& Jeglum, 2013), 对 地理环境导致的植物自身内在的化学品质差异关注 不够。我们沿纬度梯度选择3处泥炭地, 以三地的植 物为分解材料, 以长白山哈泥泥炭地为实验地, 开 展植物残体分解研究, 尝试回答泥炭地不同地理来 源植物类群的残体分解差异及其与化学品质之间的 关系，具体验证：1)是否来自低纬度地区的植物残 体具有更高的分解速率; 2)植物残体分解速率的不 同是否是植物残体的初始化学属性差异决定的。

\section{1 材料和方法}

\section{1 材料来源地与实验地概况}

本研究中, 沿纬度梯度, 选择湖北神农架大九 湖泥炭地 $\left(31.48^{\circ} \mathrm{N}, 109.98^{\circ} \mathrm{E}\right)$ 、吉林长白山哈泥泥 炭地 $\left(42.20^{\circ} \mathrm{N}, 126.52^{\circ} \mathrm{E}\right)$ 和内蒙古大兴安岭满归泥 炭地 $\left(52.03^{\circ} \mathrm{N}, 122.05^{\circ} \mathrm{E}\right)$ 为取样地点。3处泥炭地分 别位于亚热带、中温带和寒温带, 在区域内均具有 代表性和典型性, 且均属于近原始状态的大型泥炭 地，以泥炭藓属(Sphagnum)植物为优势植物，发育一 定厚度的藓类泥炭层。实验样品埋藏地哈泥泥炭地 
地处湿润半湿润针阔叶林带, 气候为中温带大陆性 季风气候, 年降水量757-930 mm (Bu et al., 2011b)。 依据2014-2017年3年的实地监测, 大九湖、哈泥和满 归 3 处泥炭地年平均气温分别为 $7.9 、 2.0$ 和 $-1.1{ }^{\circ} \mathrm{C}$ 。

\section{2 研究材料准备}

实验材料包括泥炭藓(S. palustre)、中央泥炭藓 (S. centrale)、中位泥炭藓(S. magellanicum)、锈色泥 炭藓(S. fuscum)、签草(Carex doniana)、毛薹草 $(C$. lasiocarpa)、瘤囊薹草(C. schmidtii)、红桦(Betula albosinensis)、油桦(B. fruticose var. ruprechtiana)和 柴桦(B. fruticosa) 10 个物种, 其来源地见表 1 。将每 个实验样地的每种实验材料分别处理装入分解袋, 共11种分解袋(哈泥和满归两处泥炭地的中位泥炭 藓分别制作两种分解袋)。2014年8月, 在3处研究地 分别收集足量的泥炭藓植株和维管植物叶片作为材 料, 为减少微生境等原因造成材料差异的影响, 同 一种的植株或叶片需经充分混合后备用。室内将泥 炭藓植株上部 $(4 \mathrm{~cm})$ (Bragazza et al., 2006)和薹草叶 剪成 $2 \mathrm{~cm}$ 片段, 与油华叶一起室温下风干后, 于 $30{ }^{\circ} \mathrm{C}$ 烘箱内烘干 $48 \mathrm{~h}$ 。使用 90 目尼龙网袋制作分解 袋, 每个分解袋中装入 $2.0 \mathrm{~g}$ 分解材料。根据分解材 料的体积设定分解袋大小, 泥炭藓植物分解袋大小 为 $10 \mathrm{~cm} \times 10 \mathrm{~cm}$, 维管植物分解袋大小为 $5 \mathrm{~cm} \times$ $10 \mathrm{~cm}$ 。

\section{3 实验设计}

2014年10月初, 将来自3处泥炭地的11种(区分 来源地)植物残体分解袋埋设到长白山哈泥泥炭地。 实验总计 55 个分解袋(11种材料 $\times$ 次重复)。将分解 袋分为 5 个区组, 每个区组包含 11 种分解材料各一 个样品。在哈泥, 选择植被及水位状况最具典型性

表1 实验用植物残体的来源地与埋放地

Table 1 The sites for litter collection and litter decomposition

\begin{tabular}{|c|c|c|}
\hline $\begin{array}{l}\text { 埋放地 } \\
\text { Site for decomposition }\end{array}$ & $\begin{array}{l}\text { 来源地 } \\
\text { Site for collection }\end{array}$ & 物种 Species \\
\hline \multirow[t]{11}{*}{ 哈泥 Hani } & 大九湖 Dajiuhu & 泥炭藓 Sphagnum palustre \\
\hline & & 签草 Carex doniana \\
\hline & & 红桦 Betula albosinensis \\
\hline & 哈泥 Hani & 中央泥炭藓 S. centrale \\
\hline & & 中位泥炭藓 S. magellanicum \\
\hline & & 毛臺草 C. lasiocarpa \\
\hline & & $\begin{array}{l}\text { 油桦 B. fruticosa var. rupre- } \\
\text { chtiana }\end{array}$ \\
\hline & 满归 Mangui & 中位泥炭藓 S. magellanicum \\
\hline & & 锈色泥炭藓S. fuscum \\
\hline & & 瘤囊薹草 C. schmidtii \\
\hline & & 柴桦 B. fruticosa \\
\hline
\end{tabular}

的地段，即泥炭藓属植物的盖度在 $95 \%$ 以上，水位 埋深为20-30 cm, 相对平整的泥炭藓藓丘(或泥炭 藓密集生长区域)生境, 随机选择5个埋放点, 埋放 点之间的距离不小于 $200 \mathrm{~cm}$, 以便区分区组间的分 解样品。在每个埋设点随机均匀埋设一个区组的分 解袋。分解袋口朝上, 且袋口距泥炭藓丘表面 4-6 cm。2015年9月末, 取出全部分解袋, 清理每个 分解袋表面的杂质, 然后单独封入封口袋内, 带回 实验室 $-20{ }^{\circ} \mathrm{C}$ 冷冻保存。

\section{4 指标测量与方法}

实验之初, 每种分解材料另外准备 5 袋, 用于样 品初始化学指标的测定。实验初始和结束时分解样 品进行如下处理与分析测定:

室内清除分解袋外附着的杂质及袋内维管植物 根系后, 将植物残体置于烘箱中 $65{ }^{\circ} \mathrm{C}$ 烘干至恒质 量, 称量干质量。先将样品研磨至粉状, 然后测定各 项化学指标。使用重铬酸钾-硫酸氧化法测量全C含 量(章家恩, 2007); 不同于桦木属和薹草属植物, 泥 炭藓属植物无真正的木质素, 但含有的类木质素物 质可通过乙酰溴-分光光度法测定, 参照Straková等 (2010)的方法, 使用乙酰溴-分光光度法测量桦木属 和臺草属的木质素和泥炭藓Klason木质素(以下均 简称木质素)含量; 使用Folin-Ciocalteu法测量总酚 含量(Singleton et al., 1999); 利用全自动间断化学 分析仪(SmartChem 140, AMS-Alliance, Guidonia, Italy)测量全 $\mathrm{N}$ 含量。

各个指标的损失以(初始值-分解后值)/初始值 $\times$ $100 \%$ 计算。

\section{5 数据处理}

应用SPSS 19.0软件完成数据处理与分析, 用 GraphPad Prism 6作图。实验涉及泥炭藓属、臺草属 和桦木属3个属的植物物种, 运用单因素方差分析 法分析泥炭地植物种、属和来源地对残体初始化学 组成及其化学计量比的影响, 每个属内物种对残体 分解的影响、各个属对残体分解的影响和 3 个来源地 对残体分解的影响, 并使用Tukey检验进行多重比 较分析。因泥炭藓属物种共计 4 种, 比其他两属多 1 个物种, 且仅中位泥炭藓涉及两个来源地, 所以中 位泥炭藓不用于单因素方差分析, 而单独运用双因 素方差分析法分析物种和来源地对其分解的影响。 利用相关分析和逐步回归分析方法分析泥炭地植物 残体的初始化学组分与干质量损失率之间的关系。 
显著性水平设置为 $\alpha=0.05$ 。

\section{2 结果}

\section{1 初始化学组分}

不同属的植物残体其初始化学组分不同。泥炭 藓属的木质素含量、 $\mathrm{C} / \mathrm{N}$ 、木质素 $/ \mathrm{C}$ 、木质素 $/ \mathrm{N}$ 最
高(图1，图2)。桦木属的C、N、总酚含量、总酚/C、 总酚 $/ \mathrm{N}$ 和总酚/木质素最高。同一属中, 植物的初始 化学成分及化学计量比也多存在差异(图1, 图2)。其 中, 来自哈泥和满归泥炭地的中位泥炭藓的 $\mathrm{C} / \mathrm{N}$ 分 别为 $32.1 \pm 1.1$ 和 $45.9 \pm 2.8$ 。

当不区分种属, 仅就地理来源看, 3处来源地植

表2 泥炭地植物种、属和来源地对残体初始化学组成及其化学计量比影响的单因素方差分析

Table 2 One-way analysis of variance for the effect of species, genus and source of plants on initial chemical index and stoichiometric ratios of litters

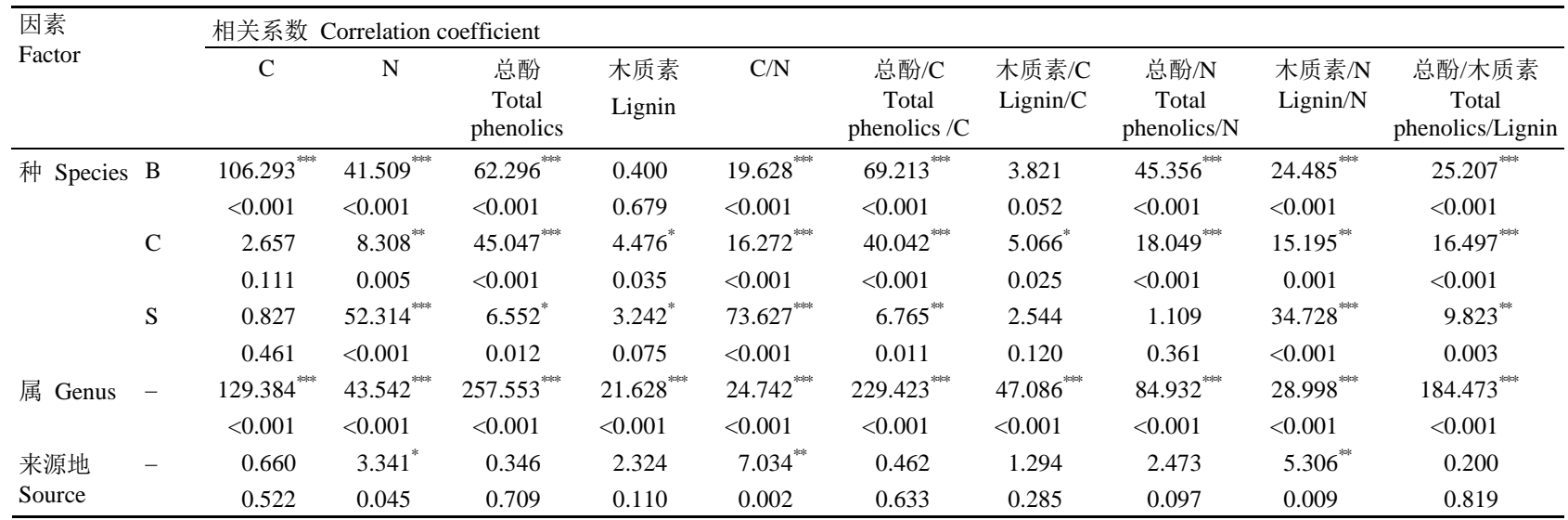

*, $p<0.05 ; * *, p<0.01 ; * * *, p<0.001$ B , 桦木属; C, 薹草属; $\mathrm{S}$, 泥炭藓属。种因素分析属内物种差异同时也是来源地差异; 来源地因素分析不同来源 地的所有植物间的差异。

B, Betula; C, Carex; S, Sphagnum. Species factor was used to analyze differences between species and also their sources. Source factor was used to analyze the differences between the averages of all the species in different sites.
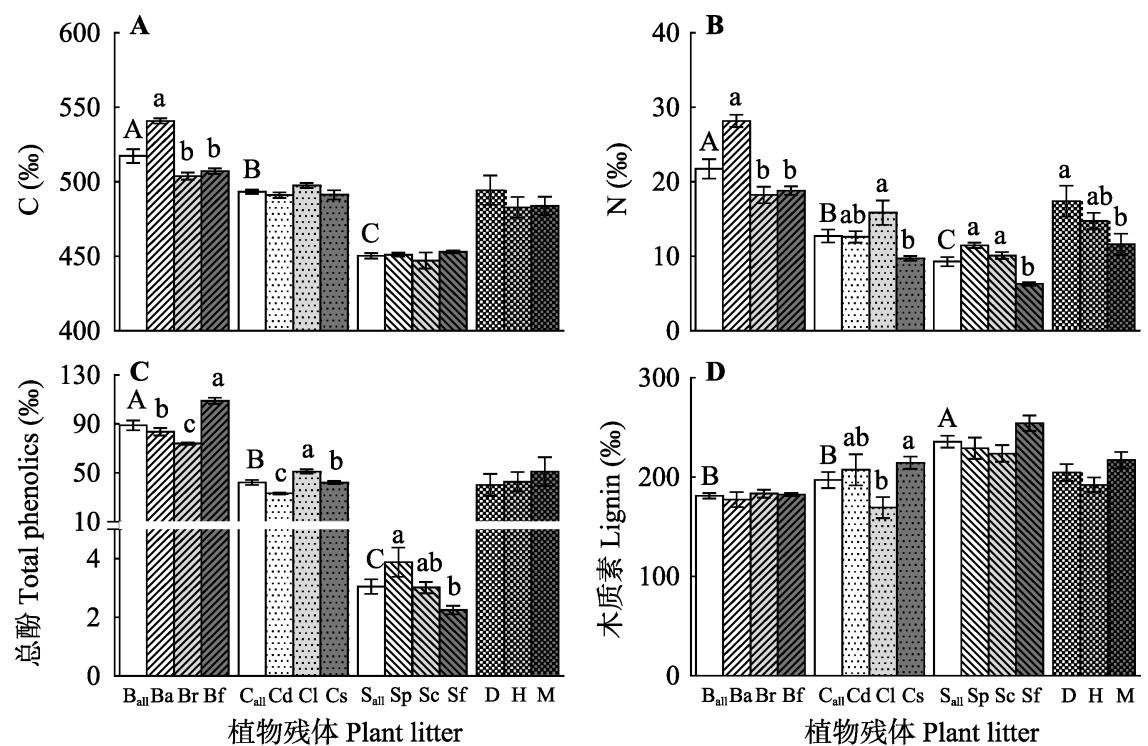

图1 泥炭地每种植物残体及每处泥炭地所有植物残体的初始化学组成(平均值土标准误差, $n=5) 。 \mathrm{~B}_{\mathrm{all}}$, 桦木属; $\mathrm{Ba}$, 红桦; $\mathrm{Br}$, 油桦; Bf, 柴桦; $\mathrm{C}_{\mathrm{all}}$, 薹草属; Cd, 签草; $\mathrm{Cl}$, 毛薹草; Cs, 瘤囊薹草; $\mathrm{S}_{\text {all }}$, 泥炭藓属; Sp, 泥炭藓; Sc, 中央泥炭藓; Sf, 锈色泥 炭藓。 $\mathrm{D}$, 来源地为大九湖的平均值; $\mathrm{H}$, 来源地为哈泥的平均值; $\mathrm{M}$, 来源地为满归的平均值。不同大写字母表示不同属之 间初始化学组成差异显著 $(p<0.05)$, 不同小写字母表示不同种和不同来源地之间初始化学组成差异显著 $(p<0.05)$ 。

Fig. 1 Initial chemical composition of each plant litter in a peatland and initial chemical composition of all the plant litters from each peatland (mean $\pm S E, n=5$ ). $\mathrm{B}_{\text {all }}$, the mean of Betula; $\mathrm{Ba}, B$. albosinensis; $\mathrm{Br}, B$. fruticosa var. ruprechtiana; $\mathrm{Bf}, B$. fruticosa; $\mathrm{C}_{\text {all, }}$, the mean of Carex; Cd, C. doniana; Cl, C. lasiocarpa; Cs, C. schmidtii; $\mathrm{S}_{\text {all, }}$, the mean of Sphagnum; Sp, S. palustre; Sc, S. centrale; Sm, S. magellanicum; Sf, S. fuscum. D, the mean of Dajiuhu; H, the mean of Hani; M, the mean of Mangui. Different capital letters indicate significant differences in initial chemical composition among genera $(p<0.05)$, and different lowercase letters indicate significant differences in initial chemical composition between both species in a genus or average of all the species among three sites $(p<0.05)$.

www.plant-ecology.com 
物残体的 $\mathrm{N}(p=0.045) 、 \mathrm{C} / \mathrm{N}(p=0.002)$ 和木质素 $/ \mathrm{N}$ $(p=0.009)$ 初始化学成分存在显著差异。大九湖泥炭 地植物残体 $\mathrm{N}$ 含量为 $(17.4 \pm 2.1) \%$, 显著高于满归 的(11.7 \pm 1.4$) \%$ o $(p=0.035)$; 哈泥的植物残体木质 素含量为(192.1 \pm 7.5)\%o, 显著低于满归的(217.0 \pm 8.4)\%。 $(p=0.091)$; C/N和木质素/ $\mathrm{N}$ 的规律相似, 满 归的数值均高于其他两地。

\section{2 植物残体分解的属、种效应}

植物残体干质量 $(p<0.001) 、 \mathrm{C}(p<0.001)$ 、总 酚 $(p<0.001)$ 和木质素 $(p=0.049)$ 的损失均因植物属 的不同而存在显著差异(表3)。桦木属和薹草属的干 质量和C损失均无显著差异(图3)。平均而言, 桦木 属和薹草属的干质量损失分别为 $(46.6 \pm 3.9) \%$ 和 $(47.0 \pm 2.2) \%$, 均超过泥炭藓属(10.3 \pm 1.8$) \%$ 的2倍
以上(图3A)。同样，桦木属 $(46.2 \pm 3.9) \%$ 和薹草属 $(46.4 \pm 2.2) \%$ 的 C损失亦远高于泥炭藓属(12.0 \pm $1.7) \%$ (图3B)。

桦木属和泥炭藓属内, 干质量损失均无显著的 物种间差异(图3)。桦木属中, 物种显著影响植物残 体木质素损失 $(p=0.031)$ 。辜草属中, 物种显著影响 植物残体干质量 $(p<0.001) 、 \mathrm{C}(p=0.001) 、 \mathrm{~N}(p<$ $0.001)$ 和总酚损失 $(p=0.006)$ 。泥炭藓属中, 物种显 著影响 $\mathrm{N}$ 损失 $(p<0.001)$ 、木质素 $(p<0.001)$ 和总酚 的损失 $(p=0.029)$ (表3)。

薹草属内, 平均而言, 芒尖薹草和毛荎草的干 质量损失分别为(52.1 \pm 2.3$) \%$ 和(51.9 \pm 1.2$) \%$, 均高 于瘤囊薹草(36.9 \pm 2.7$) \%$ (图3A), 同样, 芒尖薹草 (51.5 \pm 2.2)\%和毛薹草(51.3 \pm 1.3$) \%$ 的C损失均远高
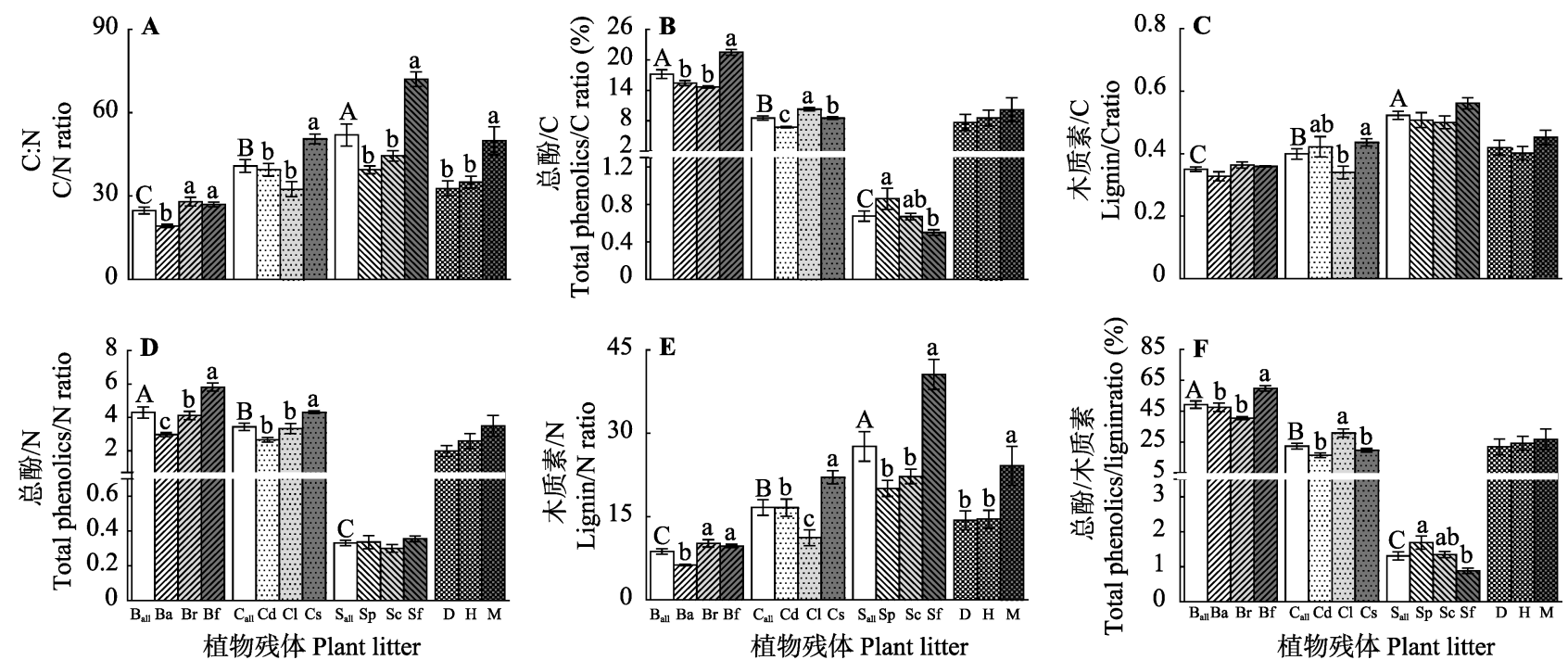

图2 泥炭地每种植物残体及每处泥炭地所有植物残体平均的初始化学计量比(平均值土标准误差, $n=5$ )。不同大写字母表示 不同属之间初始化学计量比差异显著 $(p<0.05)$ 。不同小写字母表示不同种和3处来源地之间初始化学计量比差异显著 $(p<$ 0.05)。图注同图1。

Fig. 2 Initial stoichiometric ratio of each plant litter in a peatland and average initial stoichiometric ratios of all the plant litters from each peatland (mean $\pm S E, n=5$ ). Different capital letters indicate significant differences in initial stoichiometric ratios among genera $(p<0.05)$. Different lowercase letters indicate significant differences in initial stoichiometric ratios between both species in a genus and average of all the species from three sources $(p<0.05)$. See Fig. 1 for notes.

表3 泥炭地植物种、属和来源地对残体分解影响的单因素方差分析

Table 3 One-way analysis of variance for the effect of species, genus and source of plant litters on decomposition in a peatland

\begin{tabular}{|c|c|c|c|c|c|c|c|c|c|c|c|}
\hline \multirow[t]{2}{*}{$\begin{array}{l}\text { 因素 } \\
\text { Factor }\end{array}$} & & \multicolumn{2}{|c|}{$\begin{array}{c}\text { 干质量损失 } \\
\text { Dry mass loss (\%) }\end{array}$} & \multicolumn{2}{|c|}{$\begin{array}{c}\text { C损失 } \\
\text { Carbon loss (\%) }\end{array}$} & \multicolumn{2}{|c|}{$\begin{array}{c}\text { N损失 } \\
\text { Nitrogen loss (\%) }\end{array}$} & \multicolumn{2}{|c|}{$\begin{array}{c}\text { 总酚损失 } \\
\text { Total phenolics loss (\%) }\end{array}$} & \multicolumn{2}{|c|}{$\begin{array}{c}\text { 木质素损失 } \\
\text { Lignin loss (\%) }\end{array}$} \\
\hline & & $F$ & $p$ & $F$ & $p$ & $F$ & $p$ & $F$ & $p$ & $F$ & $p$ \\
\hline \multirow[t]{3}{*}{ 种 Species } & B & 1.411 & 0.282 & 0.920 & 0.425 & 0.990 & 0.400 & 0.020 & 0.980 & $4.726^{*}$ & 0.031 \\
\hline & $\mathrm{C}$ & $16.381^{* * * *}$ & $<0.001$ & $14.883^{* *}$ & 0.001 & $15.432 * * *$ & $<0.001$ & $8.005^{* *}$ & 0.006 & 2.229 & 0.150 \\
\hline & $\mathrm{S}$ & 2.524 & 0.122 & 2.902 & 0.094 & $38.145^{* * *}$ & $<0.001$ & $4.842 *$ & 0.029 & $18.463^{* * *}$ & $<0.001$ \\
\hline 属 Genus & - & $57.069^{* * *}$ & $<0.001$ & $50.719 * * *$ & $<0.001$ & 0.387 & 0.681 & $417.741^{* * *}$ & $<0.001$ & $3.235^{*}$ & 0.049 \\
\hline 来源地 Source & - & 0.046 & 0.995 & 0.025 & 0.976 & 1.598 & 0.214 & 0.201 & 0.818 & 1.804 & 0.177 \\
\hline
\end{tabular}

*, $p<0.05 ; * *, p<0.01 ; * * *, p<0.001$ B , 桦木属; C, 臺草属; $\mathrm{S}$, 泥炭藓属。种因素分析属内物种差异同时也是来源地差异; 来源地因素分析不同来源 地的所有植物间的差异。

B, Betula; C, Carex; S, Sphagnum. Species factor was used to analyze difference between species and also its sources. Source factor was used to analyze the difference between the averages of all the species in different sites. 
于瘤囊薹草(36.4 \pm 2.9$) \%$ (图3B)。此外，芒尖薹草和 毛薹草两种薹草的 $\mathrm{N}$ 和总酚损失亦高于瘤囊薹草 (图3C, 3D)。

\section{3 植物残体分解的来源地效应}

总体上看, 来自3处泥炭地的植物残体经一年 的分解后，在干质量、C、N、总酚和木质素损失方 面均无显著差异(图3; 表3)。然而, 中位泥炭藓植物
残体干质量 $(p=0.001) 、 \mathrm{C}(p<0.001)$ 和 $\mathrm{N}(p<0.001)$ 和木质素 $(p<0.001)$ 的损失均因来源地的不同而不 同(图4)。来自哈泥的干质量损失达(19.4 \pm 1.5$) \%$ ，超 过来自满归的 $(9.0 \pm 1.5) \% 1$ 倍以上。

\section{4 初始化学组分与分解的关系}

Pearson相关分析(表4)表明，桦木属植物残体 分解率各初始化学组分无关; 臺草属植物残体的分

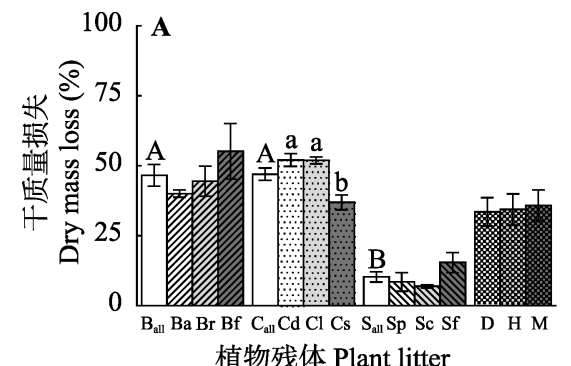
植物残体 Plant litter

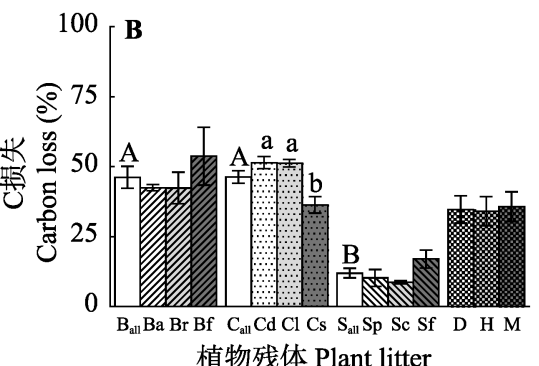

植物残体 Plant litter

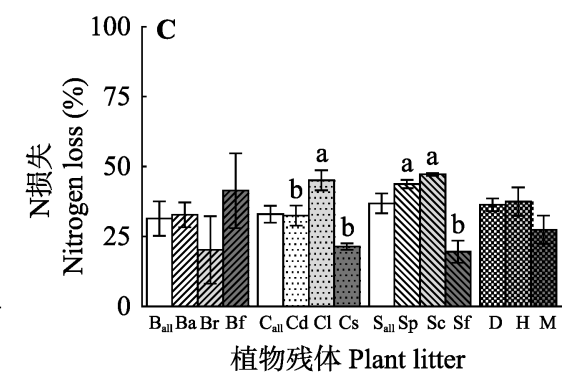

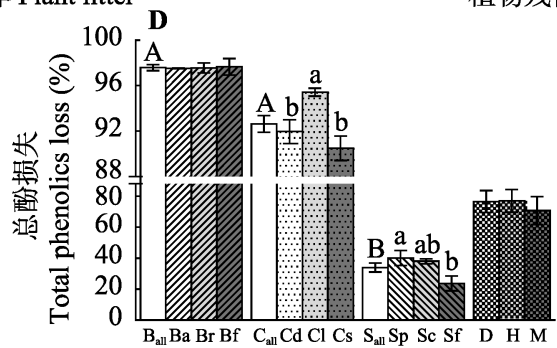

植物残体 Plant litter

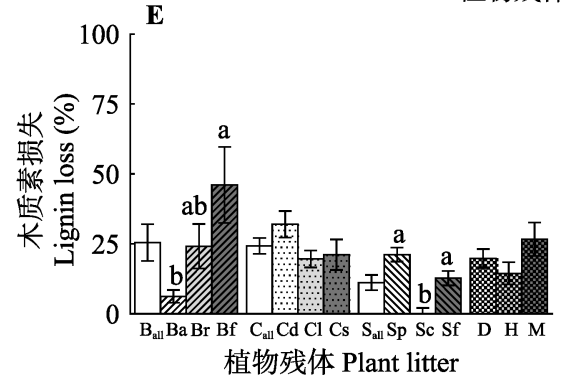

图3 物种及来源地对植物残体干质量 $(\mathbf{A}) 、 \mathrm{C}(\mathbf{B}) 、 \mathrm{~N}(\mathbf{C})$ 、总酚 $(\mathbf{D})$ 和木质素 $(\mathbf{E})$ 损失的影响(平均值土标准误差, $n=5)$ 。不同大 写字母表示不同属之间在干质量、C、N、总酚和木质素损失存在显著差异 $(p<0.05)$, 不同小写字母表示属内不同种和不同来 源地所有植物物种之间在干质量、C、N、总酚和木质素损失存在显著差异 $(p<0.05)$ 。图注同图1。

Fig. 3 The effects of species and source on losses of litter dry mass (A), C (B), N (C), total phenolics (D) and lignin (E)(mean \pm $S E, n=5)$. Different capital letters indicate significant differences in the effects of different genera on dry mass, C, N, total phenolics and lignin loss $(p<0.05)$. Different lowercase letters indicate significant differences in dry mass, C, N, total phenolics and lignin losses between different species in a same genus and among all species from different sources $(p<0.05)$. See Fig. 1 for notes.
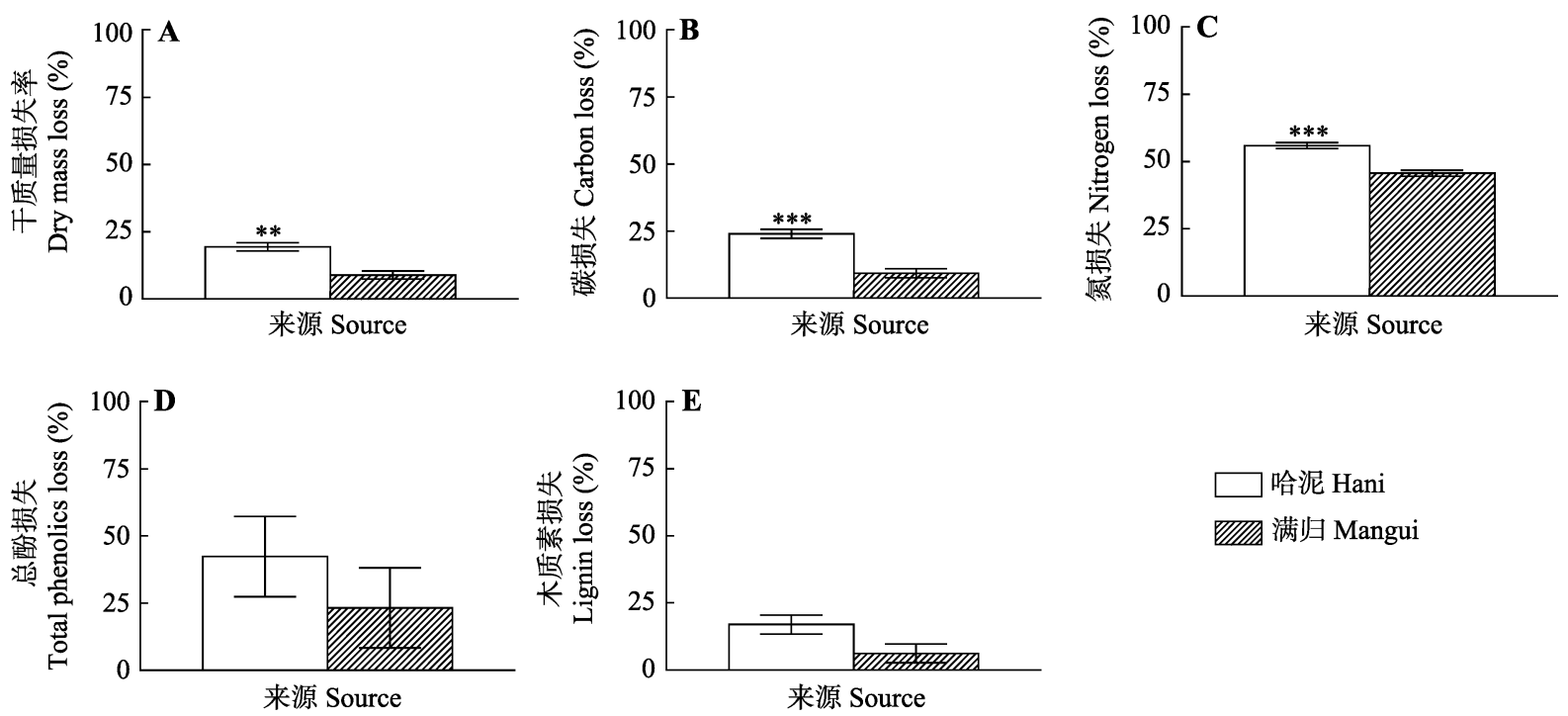

图4 来源地对中位泥炭藓植物残体干质量 $(\mathbf{A}) 、 \mathrm{C}(\mathbf{B}) 、 \mathrm{~N}(\mathbf{C})$ 、总酚 $(\mathbf{D})$ 和木质素 $(\mathbf{E})$ 损失的影响(平均值 \pm 标准误差, $n=5)$ )。*, $p<0.01 ; * * *, p<0.001$ 。

Fig. 4 Effect of plant litter source on the losses of dry mass (A), C (B), N (C), total phenolics (D) and lignin (E) of Sphagnum magellanicum litters (mean $\pm S E, n=5$ ). ${ }^{* *}, p<0.01$; ${ }^{* * *}, p<0.001$.

www.plant-ecology.com 
解率与它的初始 $\mathrm{N}$ 含量 $(p<0.01)$ 呈显著正相关关系, 与初始木质素含量 $(p<0.05) 、 \mathrm{C} / \mathrm{N}(p<0.01)$ 、木质 素/C $(p<0.05)$ 、总酚 $/ \mathrm{N}(p<0.01)$ 和木质素 $/ \mathrm{N}(p<$ $0.01)$ 呈显著负相关关系; 泥炭藓属植物残体的分解 率与初始Klason木质素含量 $(p<0.01) 、 \mathrm{C} / \mathrm{N}(p<$ $0.01)$ 、木质素 $/ \mathrm{C}(p<0.01)$ 和木质素 $/ \mathrm{N}$ 比例 $(p<0.01)$ 呈显著正相关关系。3个属总体来看, 除 $\mathrm{N} 、 \mathrm{C} / \mathrm{N}$ 和 木质素 $/ \mathrm{N}$ 外，其他指标都与干质量损失率相关。

逐步回归分析表明, C/N $\left(X_{1}\right)$ 和 $\mathrm{N}$ 含量 $\left(X_{2}\right)$ 显著 抑制臺草属植物残体分解 $\left(Y=258.354-3.088 X_{1}-\right.$ $\left.6.687 X_{2}, p<0.05\right)$, 二者可解释薹草属植物残体干 质量损失率变化的 $70 \%$ 。木质素含量 $\left(X_{3}\right)$ 显著促进泥 炭藓属植物残体分解 $\left(Y=0.276 X_{3}-54.767, p=\right.$ 0.36), 可解释干质量损失率变化的 $24 \%$ 。3个属总体 来看, 残体初始总酚 $/ \mathrm{N}\left(X_{4}\right)$ 显著促进植物残体分解 $\left(Y=11.921+8.439 X_{4}, p=0.002\right)$, 可解释干质量损失 率变化的 $72 \%$ 。

\section{3 讨论}

\section{1 植物残体分解的等级结构}

许多分解实验均揭示了残体分解的植物类群差 异性。Moore和Basiliko (2006)甚至提出, 泥炭地植 物残体分解率存在清晰的等级结构: 木本材料 $<$ 藓 丘泥炭藓 $<$ 丘间和丘坡泥炭藓 $<$ 针叶叶片 $<$ 阔叶树叶 片<灌木叶片<莎草。本研究发现, 以桦木属为代表 的灌木叶片和以臺草属为代表的莎草叶的干质量损 失均超过泥炭藓属2倍以上, 符合该分解率等级结 构, 也与Dorreapaal等(2005)结果相一致。按照该等 级结构, 丘间泥炭藓的分解率要远高于藓丘种, 如 狭叶泥炭藓(S. cuspidatum) 比锈色泥炭藓的分解速 率快1.5倍(Johnson \& Damman, 1991); 偏叶泥炭藓 (S. subsecundum) 和针叶泥炭藓( S. flexosum) 的平均
分解率(23.8\%) 高于锈色泥炭藓和尖叶泥炭藓 $(S$. capillifolium)的平均值(16.8\%)(Bragazza et al., 2007); 潒叶泥炭藓(S. fallax)的干质量损失(26.3\%)超过藓 丘种中央泥炭藓(12.1\%) 1倍以上(李伟等, 2013)。然 而, 本研究中发现, 同为藓丘种的泥炭藓属植物的 分解率却差异巨大。中位泥炭藓和锈色泥炭藓的干 质量损失比泥炭藓均高出 1 倍以上, 而且通常形成 低丘的中位泥炭藓分解速率却与形成高丘的锈色泥 炭藓相近, 此外, 灌木桦木属和薹草属叶的分解率 没有显著差异, 这些均与等级结构存在差异, 表明 Moore和Basiliko (2006) 提出的分解等级结构应仅适 用于同一甚至相近地理来源的植物泥炭地植物残体。

\section{2 生物化学属性与残体分解}

植物残体的品质是由其生物化学属性决定的。 本研究发现, 制约残体分解的因素因植物类群的不 同而不同，残体初始总酚/ $\mathrm{N}$ 是决定属间残体干质量 损失率差异的重要指标。桦木属植物的初始生物化 学属性与残体分解间完全没有关系; 葍草属和泥炭 藓属植物中, 一半左右的初始化学属性与残体分解 显著相关(表4)。这可能意味着, 薹草和泥炭藓属植 物残体的化学品质在残体分解中的决定性作用, 而 木本植物的分解方面可能更多受控于分解者或者分 解环境。来自满归泥炭地的中位泥炭藓的初始 $\mathrm{C} / \mathrm{N}$ 高于哈泥, 同时, 来自满归的锈色泥炭藓的残体具 有异于其他泥炭藓植物的突出特征, 其初始 $\mathrm{C} / \mathrm{N}$ 达 70 , 总酚/N达5\%, 分别远高于和低于其他 3 种泥炭 藓和来自哈泥泥炭地的锈色泥炭藓(分别平均为 41\%o和7\%, 未发表), 异于寻常的生物化学属性可 能是对较高纬度地区低温胁迫的一种适应响应, 也 是导致其与分解间存在异常关系的重要原因。

木质素和总酚是植物残体中难分解的物质, 通 常与植物残体分解呈负相关关系(Tahvanainen \&

表4 泥炭地植物残体干质量损失与初始化学组分的相关分析

Table 4 Correlation analysis between dry mass loss and initial chemical traits in plant litters

\begin{tabular}{|c|c|c|c|c|c|c|c|c|c|c|}
\hline & & & & & & 相关系数 Correlat & on coefficier & & & \\
\hline & $\mathrm{C}$ & $\mathrm{N}$ & $\begin{array}{c}\text { 总酚 } \\
\text { Total phenolics }\end{array}$ & $\begin{array}{l}\text { 木质素 } \\
\text { Lignin }\end{array}$ & $\mathrm{C} / \mathrm{N}$ & $\begin{array}{c}\text { 总酚/C } \\
\text { Total phenolics/C }\end{array}$ & $\begin{array}{l}\text { 木质素/C } \\
\text { Lignin/C }\end{array}$ & $\begin{array}{c}\text { 多酚/N } \\
\text { Total phenolics/N }\end{array}$ & $\begin{array}{l}\text { 木质素/N } \\
\text { Lignin/N }\end{array}$ & $\begin{array}{c}\text { 多酚/木质素 } \\
\text { Total phenolics/Lignin }\end{array}$ \\
\hline B & -0.291 & -0.301 & 0.370 & 0.264 & 0.286 & 0.404 & 0.280 & 0.433 & 0.281 & 0.345 \\
\hline $\mathrm{C}$ & 0.400 & $0.720^{* *}$ & 0.002 & $-0.526^{*}$ & $-0.787^{* *}$ & -0.014 & $-0.518^{*}$ & $-0.784^{* *}$ & $-0.740^{* *}$ & 0.251 \\
\hline S & 0.469 & -0.493 & -0.403 & $0.544^{*}$ & $0.516^{*}$ & -0.403 & $0.543^{*}$ & 0.472 & $0.523^{*}$ & -0.444 \\
\hline $\begin{array}{l}\text { 总体 } \\
\text { Total }\end{array}$ & $0.796^{*}$ & 0.531 & $0.801^{* *}$ & $-0.793^{* *}$ & -0.555 & $0.815^{* *}$ & $-0.840^{* *}$ & $0.870^{* *}$ & -0.655 & $0.793^{*}$ \\
\hline
\end{tabular}


Haraguchi, 2013)。然而, 本研究中, Pearson相关分析 表明, 泥炭藓残体分解竟然因这些难分解化学物质 含量的增加而增加, 进一步的逐步回归也表明, 初 始Klason木质素含量越高, 泥炭藓属植物残体分解 将越快, 这应与锈色泥炭藓独特的化学属性及分解 特征有关(当剔除该物种时, 这些相关关系会消失)。 同样, 当将3种生活型植物总体分析时, 不仅未发现 总酚/N (Bragazza et al., 2007)或木质素/N (Moore et $a l ., 2005)$ 对残体干质量损失的负效应, 还发现C、总 酚、总酚/N指标都与分解呈正相关关系。这种异于 常识的结果可能是本研究材料使用多种生活型材料 造成的。其中涉及的总酚本身是混合物, 不同类群 甚至物种都可能在总酚的组成上存在差异; 总酚的 积累可能是厌氧条件下氧化酶活性低导致的(Tahvanainen \& Haraguchi 2013; Medvedeff et al., 2015), 一旦所有材料放置于厌氧水平一致的环境中, 这种 原有差异可能会发生改变从而影响分解, 而泥炭藓 的Klason木质素非严格意义的木质素, 因此也可能 影响我们对木质素和分解关系的认识。

\section{3 残体分解的来源地效应}

本研究中发现, 若不考虑物种及生活型差异, 地理来源对残体分解无影响; 若选择来自两地的同 一物种比较来看, 来自哈泥的中位泥炭藓的分解远 大于来自满归的, 进而说明物种差异将极大影响残 体分解纬度梯度格局。本研究中, 同一物种的来源 地效应一方面原因是, 地理纬度环境的差异可影响 残体的品质, 高纬度地区的植物残体往往比低纬度 地区的植物残体分解更慢, 同样, 薹草属内, 来源 于高纬度寒温带地区的瘤囊薹草较来自亚热带的签 草和温带的毛臺草的干质量损失率均低许多; 另一 方面原因可能是植物残体分解的家域优势 (home field advantage), 即专属分解者的高效性导致植物 残体往往在其来源地表现出更快的分解速率(Ayres et al., 2009)。这一点在最新的泥炭地分解试验研究 中也得到了证实, 无论泥炭藓还是喜草均在其为优 势植物的生境中表现出明显的家域优势(Palozzi \& Lindo, 2017)。

\section{4 气候变化与碳累积}

在纬度梯度上, 当仅考虑以泥炭藓为重要成分 的贫营养型泥炭地时, 在北半球, 从亚热带至亚北 极, 泥炭地的面积随纬度增加呈现以 $53^{\circ} \mathrm{N}$ 为峰值 的单峰型曲线变化规律(Carvalhais et al., 2014)。泥
炭累积是净初级生产力大于分解作用的结果。在纬 度梯度上, 随纬度增加, 植物残体分解可呈现下降 趋势(白光润等, 2004; Breeuwer et al., 2008)。然而, 多年来气候变暖 $(\mathrm{Bu}$ et al., 2011a; Wang et al., 2015)、N沉降等(Bragazza et al., 2006; Bubier et al., 2007; 曾竞等, 2013)一定程度改变了泥炭地冷、湿 润、贫营养等环境特征, 造成许多北方泥炭地中维 管植物如小灌木甚至草本植物优势度增加。本研究 中, 桦木属、臺草属和铁木属等维管植物的分解率 远大于泥炭藓属植物, 表明泥炭地中树木和草本植 物的数量增加会使分解加快, 可能不利于碳积累。 当以分解材料自寒温带移置于中温带反映气候变暖 对植物残体分解的影响时, 可以看出, 短期气候变 暖对植物残体分解的影响可能较弱; 长期气候变暖 通过影响植物的生物化学属性(如中位泥炭藓), 将 大大提高植物残体的分解, 降低泥炭地的碳累积功 能。当然, 目前我们关于未来泥炭地的残体分解方 面的认识, 主要都是基于单一类型残体分解实验而 获得的。事实上, 泥炭地的植物残体分解往往是不 同残体在混合条件下进行的, 植物多样性(Gogo et al., 2016; Palozzi \& Lindo, 2017)、组成(Coq et al., 2011)等对于残体分解均可能产生影响, 因此分解 作用以及碳累积可能很复杂, 有明显的不确定性。

综上所述, 来自 3 地 10 种植物残体经过一年的 分解后, 不同属植物残体分解率(干质量损失率)差 异巨大。在同一环境条件下, 来自不同地理来源植 物残体(不考虑生活型)总体上无差异, 相同植物(中 位泥炭藓)则存在明显地理来源效应, 高纬度地区 的植物残体分解缓慢。制约残体分解的因素因植物 类群而不同, 残体初始总酚 $/ \mathrm{N}$ 是决定不同生活型 (属)间残体干质量损失率差异的重要指标。华木属 植物的初始化学属性与残体分解无关, 而臺草属植 物初始 $\mathrm{N}$ 含量和 $\mathrm{C} / \mathrm{N}$ 与残体分解、泥炭藓属植物初始 Klason木质素含量和总酚 $/ \mathrm{N}$ 与植物残体分解均呈正 相关关系。综合来看, 长期的气候变暖可能通过改 变高纬度泥炭地植物的生物化学属性, 来改变植物 残体分解, 进而影响泥炭地的碳汇功能。

致谢 感谢东北师范大学陈永达参与了野外样品的 处理。

\section{参考文献}

Aerts R (1997). Climate, leaf litter chemistry and leaf litter 
decomposition in terrestrial ecosystems: A triangular relationship. Oikos, 79, 439-449.

Ayres E, Steltzer H, Simmons BL, Simpson RT, Steinweg JM, Wallenstein MD, Mellor N, Parton WJ, Moore JC, Wall DH (2009). Home-field advantage accelerates leaf litter decomposition in forests. Soil Biology \& Biochemistry, 41, 606-610.

Bai GR, Wang SZ, Gao J, Yu JL (2004). Liquid-heat conditions and microbic decomposition on the forming of turf deposits. Journal of Shanghai Normal University (Natural Sciences), 33(3), 91-97. [白光润, 王淑珍, 高峻, 于金莲 (2004). 中国亚热带、热带泥炭形成的水热条件与微生 物分解相关性. 上海师范大学学报(自然科学版), 33(3), 91-97.]

Berg B, Berg MP, Bottner P, Box E, Breymeyer A, Anta RCD, Couteaux M, Escudero A, Gallardo A, Kratz WR, Madeira M, Mälkönen E, McClaugherty CA, Meentemeyer V, Muñoz F, Piussi P, Remacle JA, Santo AVD (1993). Litter mass loss rates in pine forest of Europe and Eastern United States: Some relationships with climate and litter quality. Biogeochemistry, 20(3), 127-159.

Bragazza L, Freeman C, Jones T, Rydin H, Limpens J, Fenner N, Ellis T, Gerdol R, Hájek M, Hájek T, Iacumin P, Kutnar L, Tahvanainen T, Toberman H (2006). Atmospheric nitrogen deposition promotes carbon loss from peat bogs. Proceedings of the National Academy of Sciences of the United States of America, 103, 19386-19389.

Bragazza L, Siffi C, Iacumin P, Gerdol R (2007). Mass loss and nutrient release during litter decay in peatland: The role of microbial adaptability to litter chemistry. Soil Biology \& Biochemistry, 39, 257-267.

Breeuwer A, Heijmans M, Robroek BJM, Limpens J, Berendse F (2008). The effect of increased temperature and nitrogen deposition on decomposition in bogs. Oikos, 117, 12581268.

Bubier JL, Moore TR, Bledzki LA (2007). Effects of nutrient addition on vegetation and carbon cycling in an ombrotrophic bog. Global Change Biology, 13, 1168-1186.

Bu ZJ, Joosten H, Li HK, Zhao GL, Zheng XX, Ma JZ, Zeng J (2011a). The response of peatlands to climate warming: A review. Acta Ecologica Sinica, 31, 157-162.

Bu ZJ, Rydin H, Chen X (2011b). Direct and interaction-mediated effects of environmental changes on peatland bryophytes. Oecologia, 166, 555-563.

Carvalhais N, Forkel M, Khomik M, Bellarby J, Jung M, Migliavacca M, Mu M, Saatchi S, Santoro M, Thurner M, Weber U, Ahrens B, Beer C, Cescatti A, Randerson JT, Reichstein M (2014). Global covariation of carbon turnover times with climate in terrestrial ecosystems. Nature, 514, 213-217.

Chen YH, Han WX, Tang LY, Tang ZY, Fang JY (2013). Leaf nitrogen and phosphorus concentrations of woody plants differ in responses to climate, soil and plant growth form. Ecography, 36, 178-184.

Coq S, Weigel J, Butenschoen O, Bonal D, Hättenschwiler S (2011). Litter composition rather than plant presence affects decomposition of tropical litter mixtures. Plant and Soil, 343, 273-286.

Dorrepaal E, Cornelissen JHC, Aerts R, Wallén B, van Logtestijn RSP (2005). Are growth forms consistent predictors of leaf litter quality and decomposability across peatlands along a latitudinal gradient? Journal of Ecology, 93, 817828.

Dyer ML, Meentemeyer V, Berg B (1990). Apparent controls of mass loss rate of leaf litter on a regional scale. Scandinavian Journal of Forest Research, 5, 311-323.

Gogo S, Laggoun-Défarge F, Merzouki F, Mounier S, Guirimand-Dufour A, Jozja N, Huguet A, Delarue F, Défarge C (2016). In situ and laboratory non-additive litter mixture effect on C dynamics of Sphagnum rubellum and Molinia caerulea litters. Journal of Soils \& Sediments, 16, 13-27.

Hobbie SE (2008). Nitrogen effects on decomposition: A five-year experiment in eight temperate sites. Ecology, 89, 2633-2644.

Irons III JG, Oswood MW, Stout RJ, Pringle CM (1994). Latitudinal patterns in leaf litter breakdown: Is temperature really important? Freshwater Biology, 32, 401-411.

Johnson LC, Damman AWH (1993). Decay and its regulation in Sphagnum peatlands. Advances in Bryology, 5, 249-296.

Johnson LC, Damman AWH (1991). Species-controlled Sphagnum decay on a South Swedish raised bog. Oikos, 61, 234-242.

Körner C (1989). The nutritional status of plants from high altitudes. Oecologia, 81, 379-391.

Li W, Bu ZJ, Zhang BJ, Long C, Tang RJ, Cui QW (2013). Decomposition of Sphagnum litter in 4 peatlands of the Changbai Mountains along an altitudinal gradient. Journal of Mountain Science, 31, 442-447. [李伟, 卜兆君, 张兵 将, 龙川, 唐瑞江, 崔钱王 (2013). 长白山不同海拔泥 炭地泥炭藓残体的分解. 山地学报, 31, 442-447.]

Medvedeff CA, Bridgham SD, Pfeifer-Meister L, Keller JK (2015). Can Sphagnum leachate chemistry explain differences in anaerobic decomposition in peatlands? Soil Biology \& Biochemistry, 86, 34-41.

Melillo JM, Aber JD, Muratore JF (1982). Nitrogen and lignin control of hardwood leaf litter decomposition dynamics. Ecology, 63, 621-626.

Moore TR, Basiliko N (2006). Decomposition in boreal peatlands. In: Wieder RK, Vitt DH eds. Boreal Peatland Ecosystems. Springer-Verlag, Berlin.

Moore TR, Trofymow JA, Siltanen M, Prescott C, Group CW (2005). Patterns of decomposition and carbon, nitrogen, 
and phosphorus dynamic. Canadian Journal of Forest Research, 35, 133-142.

Müller T, Magid J, Jensen LS, Nielsen NE (2003). Decomposition of plant residues of different quality in soil-DAISY model calibration and simulation based on experimental data. Ecological Modelling, 166, 3-18.

Ouyang LM, Wang C, Wang WQ, Tong C (2013). Carbon, nitrogen and phosphorus stoichiometric characteristics during the decomposition of Spartina alterniflora and $C y$ perus malaccensis var. brevifolius litters. Acta Ecologica Sinica, 33, 389-394. [欧阳林梅, 王纯, 王维奇, 全川 (2013). 互花米草与短叶茫芏枯落物分解过程中碳氮磷 化学计量学特征. 生态学报, 33, 389-394.]

Palozzi JE, Lindo Z (2017). Pure and mixed litters of Sphagnum and Carex exhibit a home-field advantage in Boreal peatlands. Soil Biology \& Biochemistry, 115, 161-168.

Rydin H, Jeglum JK (2013). The Biology of Peatlands. Oxford University Press, Oxford.

Singleton VL, Orthofer R, Lamuela-Raventós RM (1999). Analysis of total phenols and other oxidation substrates and antioxidants by means of folin-ciocalteu reagent. Methods in Enzymology, 299, 152-178.

Straková P, Anttila J, Spetz P, Kitunen V, Tapanila T, Laiho R (2010). Litter quality and its response to water level drawdown in boreal peatlands at plant species and community level. Plant and Soil, 335, 501-520.

Stubbs TL, Kennedy AC, Reisenauer PE, Burns JW (2009). Chemical composition of residue from cereal crops and cultivars in dryland ecosystems. Agronomy Journal, 101, 538-545.

Tahvanainen T, Haraguchi A (2013). Effect of pH on phenol oxidase activity on decaying Sphagnum mosses. European Journal of Soil Biology, 54, 41-47.

Wang HJ, Richardson CJ, Ho MC (2015). Dual controls on carbon loss during drought in peatlands. Nature Climate Change, 5, 584-587.

Wang H, Yan PF, Zhan PF, Zhang XN, Liu ZY, Guo YJ, Xiao DR (2018). The relative contributions of litter quality, simulated rising temperature, and habitat to litter decomposition. Chinese Journal of Applied Ecology, 29, 474-482. [王行, 间鹏飞, 展鹏飞, 张晓宁, 刘振亚, 郭 玉静, 肖德荣 (2018). 调落物植物质量、模拟增温及生 境对调落物分解的相对贡献. 应用生态学报, 29, 474482.]

Wang J, Huang JH (2001). Comparison of major nutrient release patterns in leaf litter decomposition in warm temperate zone of China. Acta Phytoecologica Sinica, 25, 375-380. [王瑾, 黄建辉 (2001). 暖温带地区主要树种 叶片调落物分解过程中主要元素释放的比较. 植物生 态学报, 25, 375-380.]

Zeng J, Bu ZJ, Wang M, Ma JZ, Zhao HY, Li HK, Wang SZ (2013). Effects of nitrogen deposition on peatland: A review. Chinese Journal of Ecology, 32, 473-481. [曾竞, 卜 兆君, 王猛, 马进泽, 赵红艳, 李鸿凯, 王升忠 (2013). 氮沉降对泥炭地影响的研究进展. 生态学杂志, 32, 473-481.]

Zhang JE (2007). Commonly Used Experimental Research Methods and Techniques in Ecology. Chemical Industry Press, Beijing. [章家恩 (2007). 生态学常用实验研究方 法与技术. 化学工业出版社, 北京.]

Zhang LH, Zhang SJ, Ye GF, Shao HB, Lin GH, Brestic M (2013). Changes of tannin and nutrients during decomposition of branchlets of Casuarina equisetifolia plantation in subtropical coastal areas of China. Plant Soil \& Environment, 59, 74-79.

特邀编委: 代力民 责任编辑: 李 敏 